\title{
JAK out of the box: myeloproliferative neoplasms-associated JAK2 V617F mutations contribute to aortic aneurysms
}

\section{Shannon E. Elf}

The Ben May Department for Cancer Research, The University of Chicago, Chicago, IL, USA

E-mail: SHANNON ELF - shannonelf@uchicago.edu

doi:10.3324/haematol.2020.277111

M yeloproliferative neoplasms (MPN) are clonal disorders of hematopoiesis arising in the hematopoietic stem cell (HSC) compartment and characterized by the excess production of mature myeloid cells. ${ }^{1}$ BCR-ABL-negative MPN include polycythemia vera $(\mathrm{PV})$, essential thrombocythemia (ET), and myelofibrosis (MF). PV is characterized by uncontrolled red blood cell production; ET, by megakaryocytic hyperplasia and elevated platelet counts; and MF, by megakaryocytic hyperplasia and bone marrow fibrosis. The molecular basis of MPN remained unknown until 2005, when four different groups described a point mutation in the pseudokinase domain of Janus kinase-2 (JAK2), a non-receptor tyrosine kinase, in the majority of MPN patients. The resulting JAK2 V617F mutant protein was found to be constitutively active, leading to hyperactive JAK-STAT signaling downstream of multiple hematopoietic cytokine receptors. ${ }^{2.5}$

Although MPN, particularly PV and ET, are commonly characterized as "indolent" diseases, patients have significantly decreased life expectancies compared to the general population. JAK2 V617F mutations are associated with increased vascular complications, which to date primarily include venous and arterial thrombosis and advanced atherosclerosis. ${ }^{6,7}$ Indeed, fatal thrombotic events represent the leading cause of death in JAK2
V617F-positive MPN patients, though the underlying mechanisms remain elusive. Mouse models of Jak2 V617F-driven MPN faithfully recapitulate this phenotype, with lethality primarily attributed to thrombosis. ${ }^{8}$

Interestingly, the prevalence of the JAK2 V617F mutation has also been found to be significantly increased in non-MPN patients with coronary artery disease and peripheral artery disease, ${ }^{9,10}$ suggesting that the JAK2 V617F mutation may play a role in additional vascular diseases. This observation is supported by the phenomenon of clonal hematopoiesis, in which clonal expansion of hematopoietic cells carrying is associated with significantly increased risk of vascular disorders. ${ }^{11}$ However, the mechanisms underlying JAK2 V617F-mediated vascular disease remain unclear, and the association of JAK2 V617F with vascular diseases outside of thrombosis, atherosclerosis, coronary artery disease and peripheral arterial disease has yet to be studied.

In this issue of Haematologica, Yokokawa et al. ${ }^{12}$ investigate the contribution of bone marrow (BM)-derived $J A K 2$ V617F to the development of aortic aneurysms (AA), a vascular disease not yet studied in MPN patients. AA often progress asymptomatically and can lead to sudden death, so understanding the mechanisms underlying their onset and associated risk factors is critically impor-

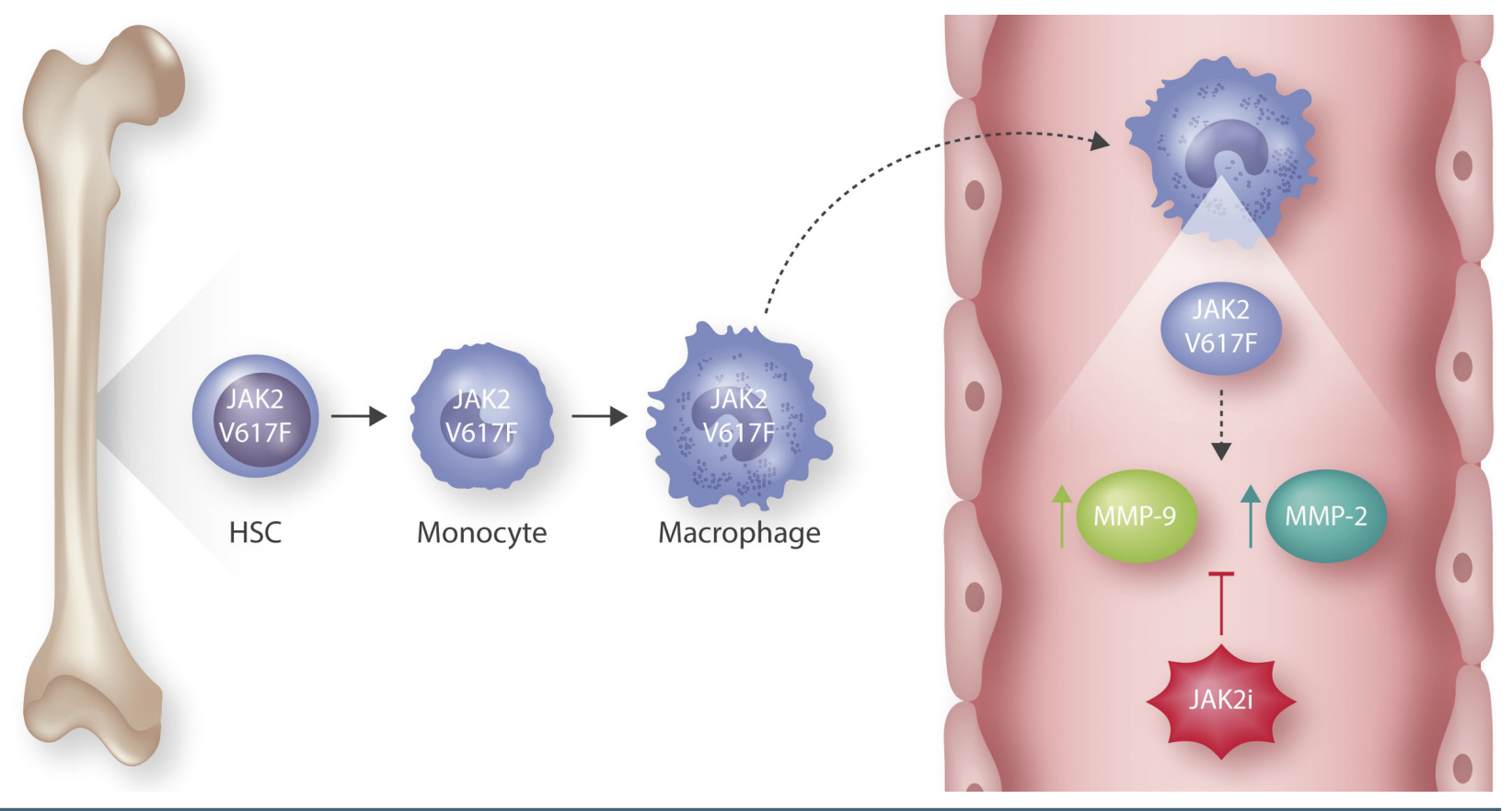

Figure 1. Hematopoietic JAK2 V617F mutations lead to the development of abdominal aortic aneurysms (AAA). Somatic JAK2 V617F mutations are acquired in the bone marrow at the hematopoietic stem cell (HSC) level. The resulting bone marrow-derived JAK2 V617F-mutated macrophages infiltrate the abdominal aorta, where they demonstrate increased levels of two genes critical for aortic aneurysm formation, matrix metalloproteins 2 and 9 (MMP-2, MMP-9). This expression and the resulting AAA are decreased upon treatment with a JAK inhibitor (JAK2i), ruxolitinib, confirming that the development of AAA is mediated by JAK2 V617F. 
tant. Here, the authors perform a prevalence study in 39 JAK2 V617F-positive MPN patients, and found that 23\% displayed signs of AA. Intriguingly, they find that JAK2 V617-positive circulating leukocytes demonstrated upregulation of genes associated with $\mathrm{AA}$, including matrix metalloproteinase 9 (MMP-9), which plays a well-established role in AA. This finding provided the authors' first insight into the potential mechanism underlying JAK2 V617F-mediated AA.

In order to further understand the role that hematopoietic-derived JAK2 V617F plays in the development of AA, the authors turn to a well characterized bone marrow transplantation (BMT) model of JAK2 V617F-driven MPN, in which JAK2 V617F expression is restricted to donor BM cells. Here, the authors utilize mice deficient in apolipoprotein $\mathrm{E}\left(\mathrm{ApoE}^{-1}\right)$ as recipients, and subject them to continuous infusion of angiotensin II (AngII), a model that has been shown to promote development and expansion of AA. In their endpoint analysis, the authors compare animals receiving wild-type (WT) versus JAK2 V617F-expressing BM cells, and find that, in addition to the expected MPN-like phenotype, JAK2 V617F BMT mice exhibit significantly increased abdominal aorta diameter, indicative of abdominal AA (AAA). Moreover, they find that JAK2 V617F expression accelerated the AAA hallmark of arterial extracellular matrix proteolysis as measured by aortic elastic lamina degradation, and led to activation of MMP-9 as well as MMP-2 in the abdominal aorta, both of which are required to produce AAA. Together, these results suggest that BM-derived JAK2 V617F promotes the development of AAA.

Digging deeper into the molecular mechanism underlying how JAK2 V617F leads to the development of AAA, the authors find infiltration of JAK2 V617F-mutant inflammatory cells, including CD68+ macrophages and Ly6B.2+ neutrophils, as well as increased phosphorylation of JAK2 V617F target STAT3, in the abdominal aortas of JAK2 V617F BMT mice receiving AngII. They go on to show that the inflammatory cells are strictly BM-derived, confirming that it is indeed hematopoietic JAK2 V617F causing the development of AAA in these animals. Finally and most intriguingly, they show that JAK2 V617F BM-derived CD68+ macrophages exhibit significantly increased mRNA expression levels of $M m p 2$ and $M m p 9$, both of which could be decreased upon treatment with the JAK2 inhibitor ruxolitinib. Additionally, AngII-treated JAK2
V617F BMT mice treated with ruxolitinib experienced decreased incidence of AAA. These data suggest that JAK2 V617F promotes activation of MMP in BM-derived inflammatory cells, which leads to AAA development (Figure 1).

Taken together, the results from this study identify a novel vascular disorder associated with JAK2 V617F mutations, provide a direct link between JAK2 V617F and the pathogenesis of AAA, and offer an additional therapeutic application for Food and Drug Admionstrationapproved JAK2 inhibitors in the prevention of AAA development in JAK2 V617F-positive MPN patients.

\section{Disclosures}

No conflicts of interest to disclose.

\section{References}

1. Campbell PJ, Green AR. The myeloproliferative disorders. N Engl J Med. 2006;355(23):2452-2466.

2. Baxter EJ, Scott LM, Campbell PJ, East C, Fourouclas N, Swanton S, et al. Acquired mutation of the tyrosine kinase JAK2 in human myeloproliferative disorders. Lancet. 2005;365(9464):1054-1061.

3. James C, Ugo V, Le Couedic JP, Staerk J, Delhommeau F, Lacout C, et al. A unique clonal JAK2 mutation leading to constitutive signalling causes polycythaemia vera. Nature. 2005;434(7037):1144-1148.

4. Kralovics R, Passamonti F, Buser AS, et al. A gain-of-function mutation of JAK2 in myeloproliferative disorders. $N$ Engl J Med. 2005;352(17):1779-1790.

5. Levine RL, Wadleigh M, Cools J, et al. Activating mutation in the tyrosine kinase JAK2 in polycythemia vera, essential thrombocythemia, and myeloid metaplasia with myelofibrosis. Cancer Cell. 2005;7(4):387-397.

6. Elliott MA, Tefferi A. Thrombosis and haemorrhage in polycythaemia vera and essential thrombocythaemia. Br J Haematol. 2005;128(3):275-290

7. Wang W, Liu W, Fidler T, et al. Macrophage inflammation, erythrophagocytosis, and accelerated atherosclerosis in Jak2 (V617F) Mice. Circ Res. 2018;123(11):e35-e47.

8. Mullally A, Lane SW, Ball B, et al. Physiological Jak2V617F expression causes a lethal myeloproliferative neoplasm with differential effects on hematopoietic stem and progenitor cells. Cancer Cell. 2010;17(6):584-596.

9. Muendlein A, Gasser K, Kinz E, et al. Evaluation of the prevalence and prospective clinical impact of the JAK2 V617F mutation in coronary patients. Am J Hematol. 2014;89(3):295-301.

10. Muendlein A, Kinz E, Gasser Ket al. Occurrence of the JAK2 V617F mutation in patients with peripheral arterial disease. Am J Hematol. 2015;90(1):E17-21.

11. Jaiswal S, Natarajan P, Silver AJ, et al. Clonal hematopoiesis and risk of atherosclerosis cardiovascular disease. N Engl J Med. 2017;377(2): 111-121.

12. Yokokawa T, Misaka T, Kimishima Y, et al. Crucial role of hematopoietic JAK2V617F in the development of aortic aneurysms. Haematologica. 2021;106(7):1910-1922. 